\title{
Effect Of Limb Rotation on Measurements Of Equine Metacarpophalangeal Radiographs
}

\section{${ }^{* 1}$ Abdulrhman M. Alrtib, ${ }^{1}$ Aiman H. Oheida, ${ }^{1}$ Mohamed H. Abushhiwa and ${ }^{2}$ Helen M.S. Davies}

\author{
${ }^{\mathbf{1}}$ Faculty of Veterinary Medicine, University of Tripoli, Tripoli 13662, Libya \\ ${ }^{2}$ The Faculty of Veterinary and Agricultural Sciences, The University of Melbourne, Parkville 3010, Australia \\ Corresponding author: Abdulrhman M. Alrtib \\ Phone: +218945110809 \\ Fax: +218214628421 \\ E-mail: A.Alrtib@uot.edu.ly \\ Address: Anatomy, Histology and Embryology department, Faculty of Veterinary Medicine, University of Tripoli, Tripoli 13662, \\ Libya
}

\section{ARTICLE HISTORY \\ Received: Novwmber 3, 2021 \\ Revised: December 2, 2021 \\ Accepted: January 16, 2022 \\ Corresponding author: Abdulrhman M. Alrtib; Phone: +218945110809; E-mail: A.Alrtib@uot.edu.ly}

\begin{abstract}
Objective: Metacarpophalangeal joint (MCPJ) radiographic parameters were developed to evaluate MCPJ conformation of equine forelimbs but the effect of joint rotation during radiography on these parameters has not been reported. The current study examined the effect of the rotation around the vertical axis of MCPJ on 27 MCPJ parameters (12 angles and 15 ratios) and identified the acceptable range of rotation at which minimal changes have occurred in these parameters.

Design: MCPJs of seven right equine forelimbs were loaded in a load cell to obtain the normal MCPJ angle. They were frozen at $-20 \mathrm{C}^{\circ}$ and then scanned using a CT-scan machine. The collected slices were reconstructed to obtain a 3D module of MCPJs, and the dorsopalmar view at zero angle (ZDP) were identified. The view was then rotated around the vertical axis of MCPJ. Snapshots were taken for MCPJ at $-10^{\circ},-5^{\circ}$, Zero, $+5^{\circ}$ and $+10^{\circ}$ angles. All the parameters were measured on the total 35 radiographs and statistically analysed. Differences were estimated per $5^{\circ}$ of MCPJ rotation for all the parameters.

Animals: MCPJs of seven right forelimbs from seven different horses with no MCPJ abnormalities.

Results: Generally, the ratio parameters were less affected than the angular parameters during MCPJ rotation.

Conclusion and clinical relevance: Rotational angles of vertical $\pm 10^{\circ}$ from ZDP were considered as an acceptable range for reliable radiographic measurements of MCPJ parameters.

Keyword: Equine, fetlock, morphometry, radiology, rotation
\end{abstract}

\section{INTRODUCTION}

Radiology employs the use of medical imaging technology to diagnose and treat many medical conditions and remains a cornerstone of the medical field. Despite radiology being the fastest and easiest way to assess bones, studying bone morphometry using conventional and digital radiographs is difficult due to the high sensitivity of this technique to any little changes during radiographing process. The alignment [1]; [2]; [3]; [4] and distances [1]; [5]; [6] between the x-ray machine, object and cassette during radiography were found to have significant effects on the radiographic measurements. Misalignment, for instance, would result in a distortion which is revealed as a lesser magnification of points that were closer to the cassette compared with those that were farther from the cassette [2]. Consequently, measurements conducted on a such distorted radiograph would misrepresent the actual size, shape and location of the object features [3].

The dorsopalmar radiograph (DP) is a $2 \mathrm{D}$ image in which the radiographic measurements would be affected by the object rotation around its vertical and horizontal axes [7]. However, rotation around the sagittal axis of the object could only change its position on DP radiographs and not on its measurements [8]. This was the reason for the sagittal axis being parallel to the centre of the X-ray beam [7].

To the best of our knowledge, the effect of rotation of the fetlock around the vertical axis on the 27 fetlock parameters which were developed by the Alrtib et al. (2019) [9] has not been investigated. Therefore, the hypothesis of the current study was that the limb rotation during radiography would have an effect on the MCPJ parameters. The present study was firstly aimed to evaluate the effect of the limb rotation around its vertical axis on MCPJ radiographic measurements. Secondly, determining an acceptable range for the limb rotation at which the parameters were subjected to the minimum amount of changes. Thirdly, establishing an equation to estimate the deviation of MCPJ vertical rotation from ZDP.

\section{MATERIALS AND METHODS}

\subsection{Animals}

Seven right distal forelimbs from seven different adult horses were used in the study. The age of the horses ranged between 12 and 21 years. All the cadaveric forelimbs were collected from the Pathology department of The University of 
Melbourne, Veterinary Science. Horses were euthanised or died for reasons that were not associated with the locomotor system.

\subsection{Preparation and scanning of the forelimbs}

The forelimbs were cut at the distal third of the radius. Each distal forelimb was prepared and installed in a load cell [10]. The installed limb was loaded until it achieved the normal MCPJ angle in a standing horse which ranged between $135^{\circ}$ and $168^{\circ}$ [11]; [12] (Figure 1). A Prestige Medical ${ }^{\circledR}$ 8-inch protractor goniometer was used to measure MCPJ angles. The distal forelimb and the load cell were put in the freezer ($\left.20 C^{\circ}\right)$. After 24 hours of freezing the limb was uninstalled from the load cell and scanned immediately using a CT-scan machine (Siemens Somatom Emotion 16 Excel EditorGermany). During the scan, the limb was placed on the moveable table of the CT-scan machine with the dorsal aspect of the limb facing up.

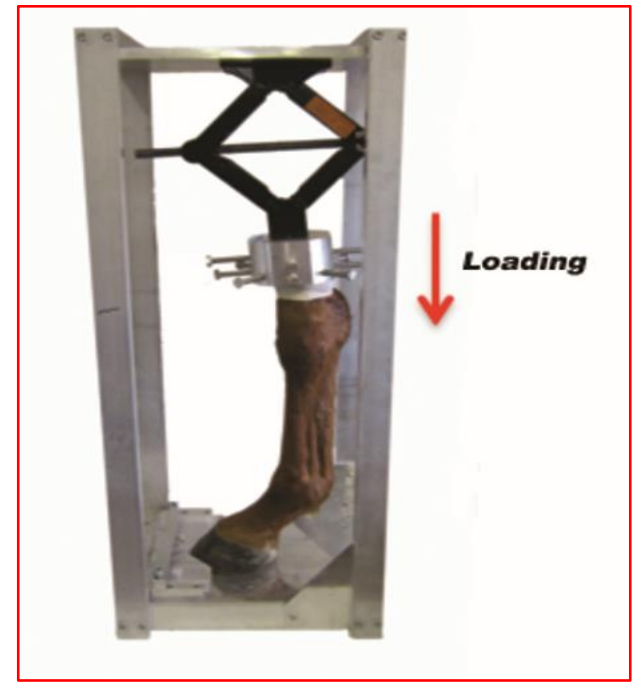

Figure 1: Installing and loading the limb in the load cell.

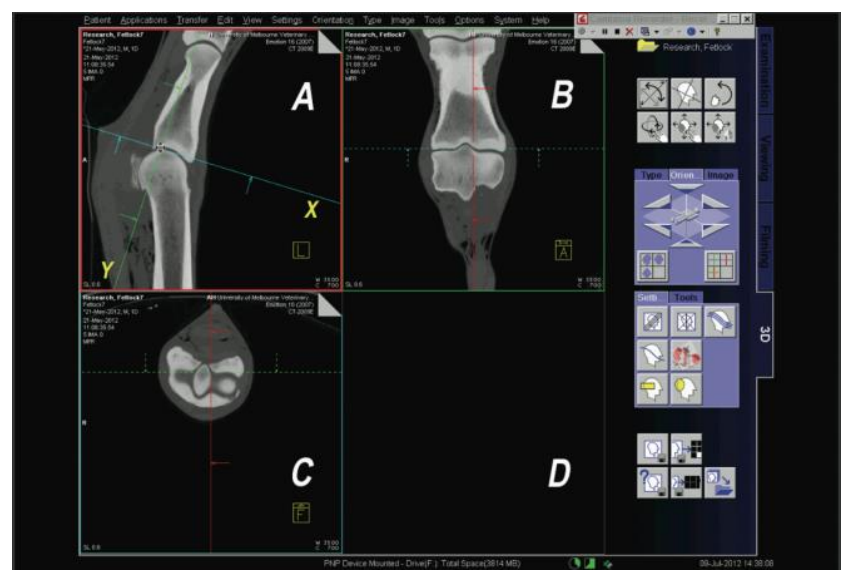

Figure 2: Screen snapshot for the software after importing the CT-slices of a right MCPJ showing (A) lateromedial slices, (B) dorsopalmar slices, (C) proximodistal slices views, and (D) screen section for the 3D model, and the rotated and moved $(\mathrm{X}) \mathrm{X}$-axis and $(\mathrm{Y}) \mathrm{Y}$-axis to obtain standard views.

\section{3. $3 D$ reconstruction of $M C P J$}

The slices of each scanned joint were imported into reconstructor software (Somaris/5 syngo CT 2009E software (Siemens AG, Medical Solutions, Germany)). The computer screen was divided into four sections including; lateromedial (A), dorsopalmar (B), and proximodistal (C) slice views for MCPJ, and another empty screen section (D) to view the 3D model after reconstruction (Figure 2). Each one of the first

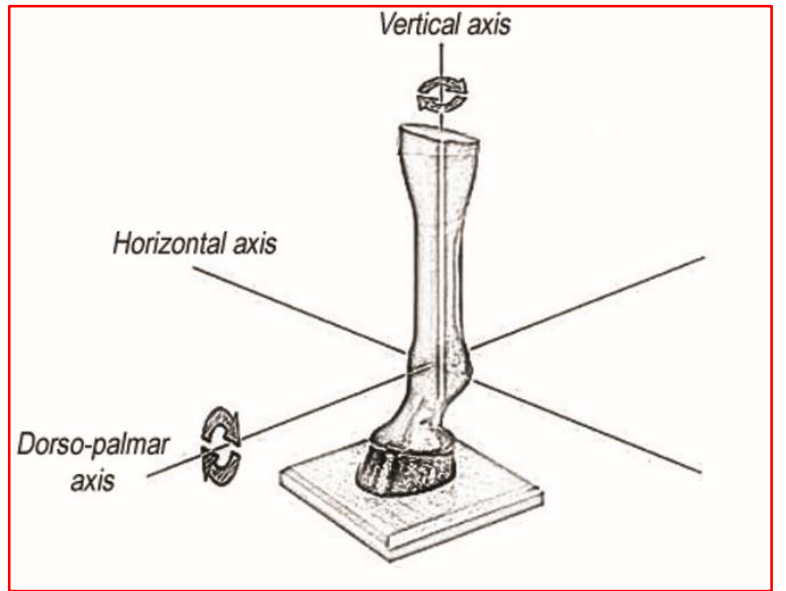

three screen sections contained two axes ( $\mathrm{X}$ and $\mathrm{Y}$ ), and these axes had a capability to be rotated and moved using the computer pointer.

Figure 3: Schematic image showing the rotation around MCPJ axes.

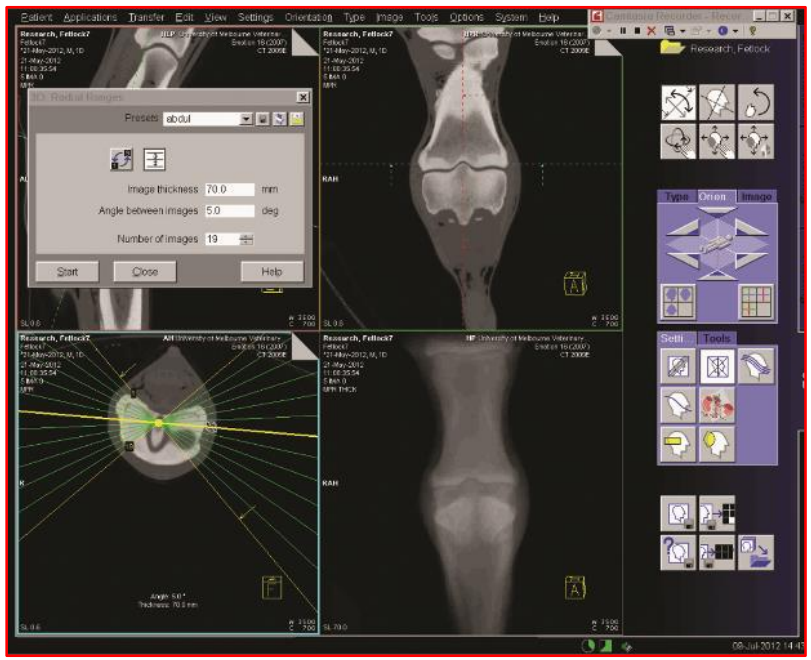

Figure 4. Screen snapshot of the software after importing the CT-slices of a right MCPJ showing how to obtain the dorsopalmar views during the rotation around the vertical axis with $5^{\circ}$ intervals

To obtain a dorsopalmar view at the zero degree (ZDP) for the MCPJ, the X-axis was assumed to represent the X-ray beam in the radiography machine and should be perpendicular to the longitudinal axis of the proximal phalanx (P1). Therefore, using the pointer, the $Y$-axis on the LM slices view was rotated to fit the longitudinal axis of $P 1$, and then the $X$-axis was rotated to be perpendicular on the $Y$-axis (Figure 2). The centre of the cross between the $X$ and $Y$-axes was then moved to be at the middle of the joint, taking into account the 
dorsopalmar thickness of the proximal sesamoid bones (PSB) which was not visible because the slice was through the intersesamoidean ligament. In the DP slices view (B), the

centre of the perpendicular axis was placed in the centre of MCPJ where the $\mathrm{X}$-axis was fitted to the longitudinal axis of $P 1$. The thickness of the 3D module obtained in this way was set depending on the joint thickness (Approximately $70 \mathrm{~mm}$ ).

\subsection{Dorsopalmar view of MCPJ rotated around its vertical} axis

The proximodistal slice view (Figure 2, C) was selected using the computer pointer and then the axis of the radial ranger tool was rotated at the middle of the joint and directed lateromedially and 19 dorsopalmar views from the rotation around the vertical axis with $5^{\circ}$ intervals were obtained (Figure 4). The rotation around the MCPJ joint axes is illustrated in figure 3.

\subsection{Collecting and preparing MCPJ radiographs for measurements}

All views obtained in this way were exported to another computer for measurement. From each limb, five views were used. These included ZDP, two positive $\left(+5^{\circ}\right.$ and $\left.+10^{\circ}\right)$ and two negative $\left(-5^{\circ}\right.$ and $\left.-10^{\circ}\right)$ rotational views. The positive (internal) and the negative (external) rotational views indicated an internal $(\mathrm{V}+)$ and external ( $\mathrm{V}$-) rotation around the vertical axis respectively. Therefore, all radiographs were labelled as V-10, V-5, Zero, V+5, and V+10 for the rotation around the vertical axis. All MCPJ parameters were measured using Hoof-Metron measurement software (Epona Tech LLC), USA.

The software has been designed to differentiate between the angle measurements depending on their directions. Hence, it deals with the right-direction angles on the screen (directed medially in the right limbs and laterally in the left limbs) as positive angles, and the left-direction angles (directed laterally in the right limbs and medially in the left limbs) as negative angles. The angles located at the medial and lateral sides were different in sign despite the values being similar on both sides. The angles with negative values can affect the calculation of the coefficient of the angle changes in the statistical analysis. Therefore, all negative angular values were changed to positive values.

\subsection{MCPJ parameters}

The 27 MCPJ parameters (12 angles and 15 ratios) used in this study were established by Alrtib et al. (2019)[9].

Angular parameters:

Base medial PSB-Proximal P1 angle (B1)

Base lateral PSB-Proximal P1 angle (B2)

Base PSBs angle (B3)

Lowest PSBs-Proximal P1 angle (B4)

Highest PSBs-Proximal P1 angle (B5)

P1 angle (B6)

Trigonum $\mathrm{P} 1$ angle (B7)

Medial trigonum-Proximal P1 angle (B8)

Lateral trigonum-Proximal P1 angle (B9)

Medial sagittal ridge Mc3- Proximal P1 angle (B11)

Lateral sagittal ridge Mc3- Proximal P1 angle (B13)
Sagittal ridge angle (B14)

\subsection{Ratio parameters}

Ratio of the lateromedial width of the medial articular cavity to the lateromedial width of the lateral articular cavity of P1 (W2/W3)

Ratio of the lateromedial width of the medial sesamoid bone to the lateromedial width of the lateral sesamoid bone (W4/W5).

Ratio of the lateromedial width of the proximal extremity of $\mathrm{P} 1$ to the lateromedial width of the distal extremity of Mc3 (W1/W6).

Ratio of the lateromedial width of the medial sesamoid bone to the palmar lateromedial width of the medial condyle of Mc3 (W4/W7).

Ratio of the lateromedial width of the medial sesamoid bone to the palmar lateromedial width ofMc3 (W4/W7+W8).

Ratio of the lateromedial width of the lateral sesamoid bone to the palmar lateromedial width of the lateral condyle of Mc3 (W5/W8).

Ratio of the lateromedial width of the lateral sesamoid bone to the palmar lateromedial width of the Mc3 (W5/W7+W8). Ratio of the palmar lateromedial width of the medial condyle to the palmar lateromedial width of the lateral condyle of Mc3 (W7/W8).

Ratio of the lateromedial width of the medial articular cavity of $\mathrm{P} 1$ to the palmar lateromedial width of the medial condyle of Mc3 (W2/W7).

Ratio of the lateromedial width of the lateral articular cavity of P1 to the palmar lateromedial width of the lateral condyle of Mc3 (W3/W8).

Ratio of the palmar lateromedial width of the medial condyle to the lateromedial width of the distal extremity of Mc3 (W7/W6).

Ratio of the palmar lateromedial width of the lateral condyle to the lateromedial width of the distal extremity of Mc3 (W8/W6).

Ratio of the proximodistal height of the medial sesamoid bone to the proximodistal height of the lateral sesamoid bone $(\mathrm{H} 1 / \mathrm{H} 2)$.

Ratio of the proximodistal height of the medial sesamoid bone to the proximodistal height of $\mathrm{P} 1(\mathrm{H} 1 / \mathrm{H} 3)$.

Ratio of the proximodistal height of the lateral sesamoid bone to the proximodistal height of $\mathrm{P} 1(\mathrm{H} 2 / \mathrm{H} 3)$.

\subsection{Statistical analysis}

A generalised linear mixed model with random effects of horse and a fixed effect of vertical angle of rotation was used to derive the regression coefficients for the effect of rotation on the parameter. Different vertical angles $\left(-10^{\circ},-5^{\circ}, 0^{\circ},+5^{\circ}\right.$ and $+10^{\circ}$ ) were used for each MCPJ. The Stata (v12.1, Stata Corp, College Station, TX) command-xtmixed-) was performed to analyse the data. A linear regression was used to estimate an association between MCPJ angle and W2/W3 ratio. Statistical significance was set at $P<0.05$.

\subsection{Acceptable Range of Rotation}


Any change less than $2^{\circ}$ in angular parameter [13]; [14] measurements and 0.2 in ratios was considered to be clinically acceptable.

\section{RESULTS}

The rotation around the vertical axis statistically showed significant changes in the 27 parameters as shown in table 1. Effect of the Rotation around the Vertical Axis of MCPJ on Parameters

Angular parameters

The external and internal rotations around the vertical axis significantly ( $p$-value $<0.001$ ) affected the values of all angular parameters except B3 and B14. The significant changes were by less than $0.73^{\circ}$ for every $5^{\circ}$ of rotation. During internal rotation, the values of $\mathrm{B} 1, \mathrm{~B} 5, \mathrm{~B} 6, \mathrm{~B} 8$ and $\mathrm{B} 11$ increased gradually, while the values of $B 2, B 4, B 7, B 9, B 13$ and B14 decreased gradually. Regardless of the direction of rotation, the greatest change in these angles was recorded in $\mathrm{B} 8$ which altered from $71.38^{\circ}$ at $-10^{\circ}$ to $74.26^{\circ}$ at $+10^{\circ}$ by $0.72^{\circ}$ per $5^{\circ}$ of rotation (Figure 5 ). Whereas the minimum angular change was recorded in the value of B14 which altered from $102.05^{\circ}$

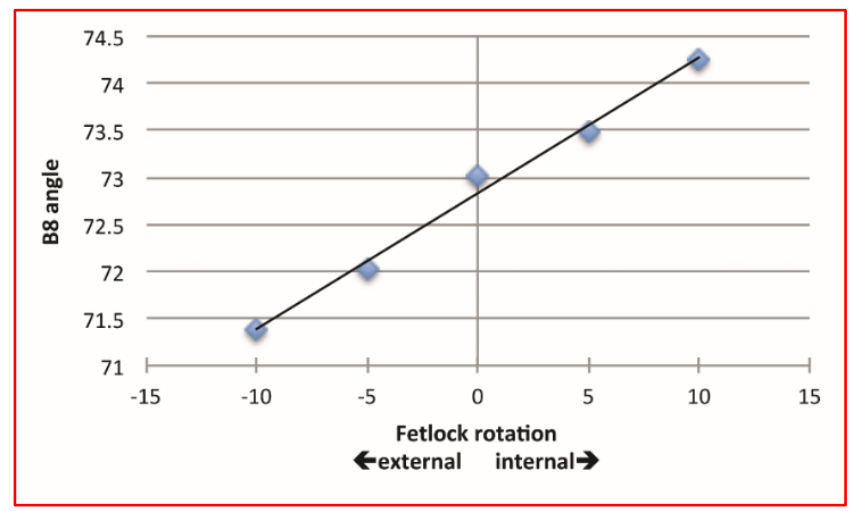

at $-10^{\circ}$ to $101.78^{\circ}$ at $+10^{\circ}$ by $0.06^{\circ}$ per $5^{\circ}$ of rotation (Figure 6).

Figure 5: Effect of rotation on B8 angle in the seven MCPJs.

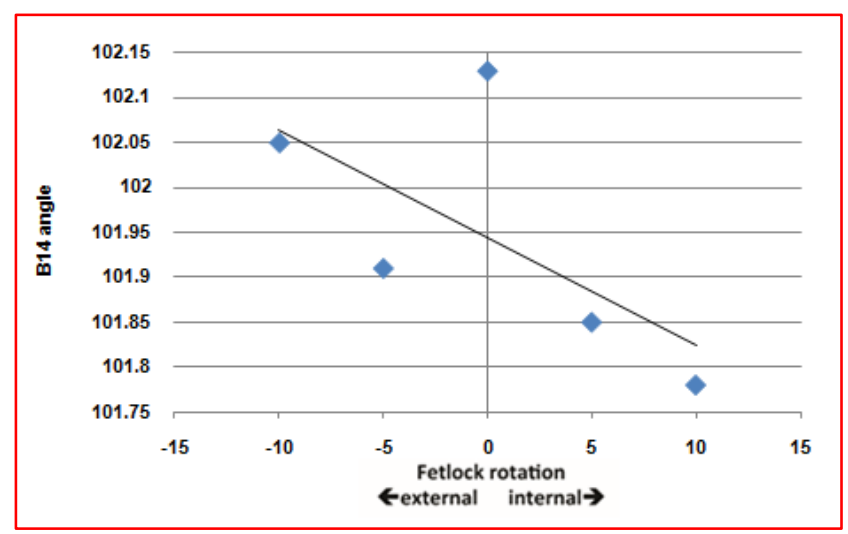

Figure 6: Effect of rotation on B14 angle in the seven MCPJs

\section{Ratios}

According to the statistics, the ratio parameters generally showed less effect from the rotation. Three of the fifteen ratios showed no significant effect of the limb rotation. They were $\mathrm{W} 1 / \mathrm{W} 6$ ratio, $\mathrm{H} 1 / \mathrm{H} 2$ ratio and $\mathrm{H} 2 / \mathrm{H} 3$ ratio. In regard to the change of the mean difference per $5^{\circ}$ of the rotation, there was a variable change among the ratios. For instance, W7/W8 altered by \pm 0.07 while W1/W6 altered by \pm 0.0006 . Despite the amount of change, it was noted that the W2/W3 ratio presented a nearly perfect linear relationship along the rotational angles (Figure 7 ).

\subsection{Acceptable range of the rotated MCPJ}

The amount of change of all parameters in every $5^{\circ}$ of the rotation around the vertical axis is summarised in table 2 . The rotation by $\pm 5^{\circ}$ demonstrated the minimal amount of change in all parameters. Overall, the angular parameters presented changes which ranged between $0.06^{\circ}$ and $0.72^{\circ}$ per $5^{\circ}$ of rotation. The minimum amount of change was found in the ratio parameters with $\leq 0.05$ per $5^{\circ}$ of rotation.

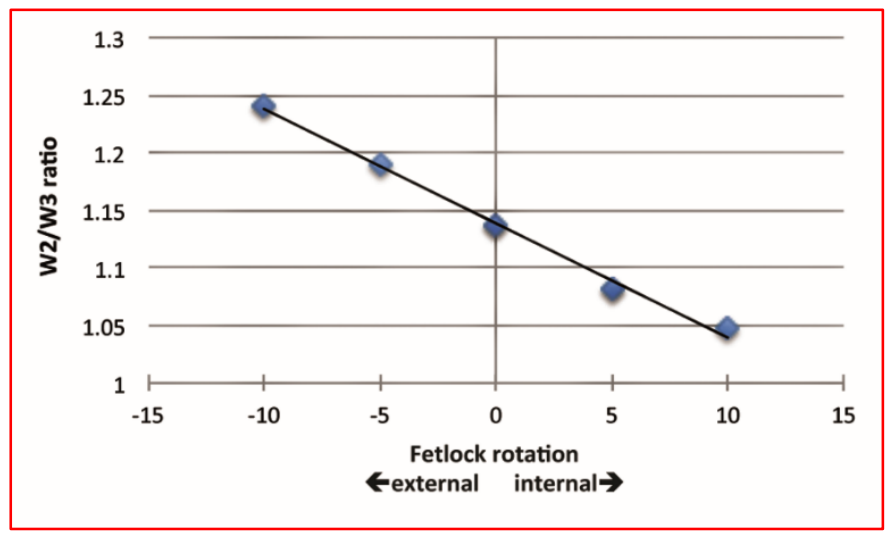

Figure 7: Analysis of the ratio W2/W3 depending on MCPJ rotation. The graph shows the decrease in the ratio during external and internal MCPJ rotation.

The W2/W3 ratio presented a near-perfect linear relationship during rotation where it altered from its maximum value (1.05) at $10^{\circ}$ to its minimum value (1.25) at $-10^{\circ}$ of the rotation around the vertical axis (Figure 7 ).

The linear regression is described by $y=-0.0099 x+1.14\left(R^{2}=\right.$ 0.99). In respect to this linear regression, any MCPJ angle change $(X)$ could be estimated with:

$$
X=(W 2 / W 3-1.14) /(-0.0099) \text { according to Figure } 8
$$

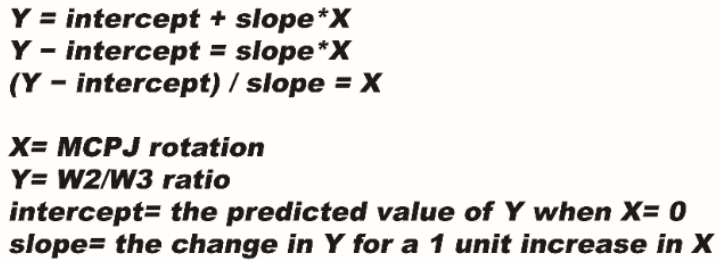

Figure 8: Equation established to estimate the degree of MCPJ rotation from the $\mathrm{W} 2 / \mathrm{W} 3$ ratio [15]

\section{Discussion}

Several landmarks on radiographs of the equine MCPJ were identified and developed [9]. However, considering the effect 
of MCPJ rotation on the developed measurements is important for field use. In this study a CT-scan was used rather than the digital $x$-ray for several reasons. The main reason was to obtain an accurate angle of rotation for MCPJ using 3D-reconstruction software, which consequently resulted in more reliable measurements. The second reason was to minimize time and cost by scanning each MCPJ once rather than taking many radiographs to achieve the five required views.

The statistical findings in this study showed that there were significant changes in most of the angular and the ratio parameters during the rotation around the vertical axis. Regardless the direction of rotation (internal or external) most of MCPJ parameters showed a linear increase or decrease in their values. This might be due to the horizontal orientation of the measured parameters. These findings were in agreement with the results of a cephalometric study which reported that the rotation around the vertical axis mostly affected the horizontal measurements [16]. Furthermore, all the vertical measurements $(\mathrm{H} 1 / \mathrm{H} 2, \mathrm{H} 1 / \mathrm{H} 3$ and $\mathrm{H} 2 / \mathrm{H} 3$ ratios) in this study did not markedly change and had approximately the same values throughout the rotations.

In contrast to the angular parameters, the ratio parameters generally had the least amount of change. This seems to be related to the small values of the ratios. Most of the parameters showed an increase or decrease in their values during internal rotation of MCPJ. These changes in the values might have occurred due to the direction (medially or laterally) of the angular parameters in regards to the vertical axis, and due to the position (medially or laterally) of the horizontal linear parameters in respect to the same plane.

In the angular parameters, the percentage of change per $5^{\circ}$ of rotation was variable. B4, B5 and B6 recorded the maximum percentage of change by more than $6.5 \%$, while the rest of the parameters showed less than $3.5 \%$ change. The potential reason for these results is the location of the landmarks of the parameters in relation to the axis of rotation. The highest percentages of change during rotation were found in the parameters where their landmarks were located on both sides of the vertical axis. In contrast, when the landmarks were only on one side of the axis of rotation their parameters showed small percentages of changes. For instance, for the B1 angle (3.11\%), both of its landmarks were located on the same side of the vertical axis (both were on either the medial or lateral side), while each landmark of the B5 angle (7.47\%) was located on different sides of the axis of rotation (Figure 9).

The evaluation of the effect of the rotation on MCPJ parameters in this study could provide a guideline to identify measurements that can be used to differentiate between different MCPJ bone conformations in racehorses. However, there were some other factors that might have a potential effect on these measurements. The orientation of the radiographic materials ( $x$-ray machine, object and the cassette) [1]; [2]; [4] and the distances between these objects [3]; [6] were considered to be the most common reasons of producing poor quality radiographs which consequently affect landmarks identification. To minimize the measurement errors that could be produced by these factors, the techniques of taking DP radiographs using an $x$-ray machine were carefully followed during reconstruction of the 3D module of MCPJ in this study.

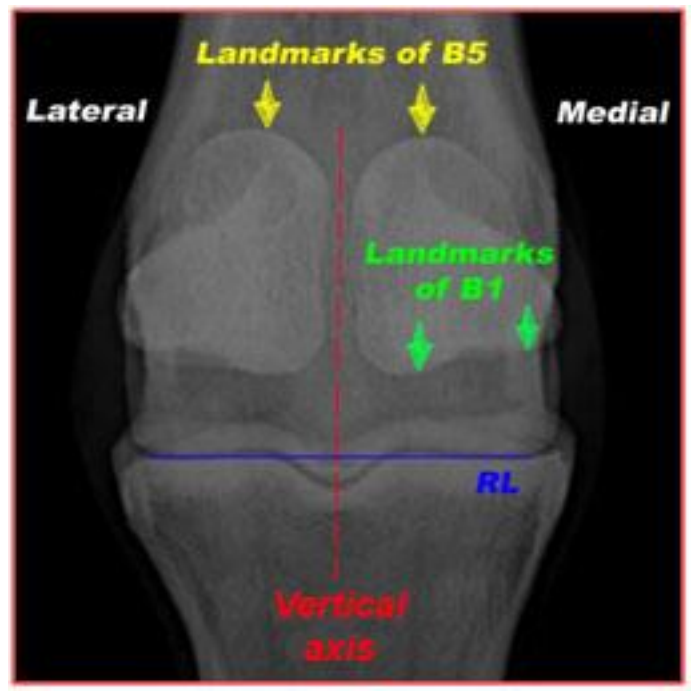

Figure 9: A dorsopalmar radiograph of right $\mathrm{MCPJ}$ showing the locations of $\mathrm{B} 1$ and B5 landmarks.

The ratio of W2/W3 in this study showed a linear trend during the rotation around the vertical axis in the DP view of MCPJ, where it decreased during internal rotation of the MCPJ. This result might be due to the location of anatomical features which were used to develop this ratio. Each of the two components of this ratio was located on different sides of the vertical axis. The W2 was located on the medial side while the W3 was located on the lateral side of the MCPJ. According to Yoon et al. (2001) [17] the further the object rotates from the film, the greater the resultant magnification. For instance, during lateral rotation around the vertical axis, W2 increased while W3 decreased, and vice versa for the medial rotation. This ratio was used to establish an equation to estimate the angle of MCPJ rotation during radiography. Such an equation offers the possibility of minimizing the errors that can be generated due to the effect of rotation using visual landmarks on the radiographs. It also helps to identify the degree of rotation of MCPJ from DP radiographs.

One of the main goals of the current study was identifying the range of the MCPJ rotation around the vertical axis at which the measurements expressed an acceptable amount of change. Other studies which investigated the effect of deviated angles on radiographic measurements have considered the measurement differences within $2^{\circ}$ in angular parameters [13]; [14]; [18] to be clinically accepted. The current results showed that the mean changes in angular measurements ranged between $0.06^{\circ}$ and $0.72^{\circ}$ per $5^{\circ}$ of the rotational angle. In the ratios, the mean change was $\leq 0.05$ in every $5^{\circ}$ of MCPJ rotation. Based on these findings, it can be 
seen that the resultant changes due to the rotation around the vertical axis are generally small not only within $5^{\circ}$ of rotation but also within $10^{\circ}$ of rotation despite being significant or not. Perhaps such little changes would be reasonable to consider the rotated MCPJ by $10^{\circ}$ around its vertical axis as an acceptable range of rotation. However, using this range as an applicable reference in the clinic obviously requires further investigation.

It can be concluded that the vertical rotation of MCPJ can significantly affect most of the parameters even though this effect produced a small change in measurements. The

Table 1: Comparison between parameter values in all MCPJ rotation angles in the $7 \mathrm{MCPJs}$. The percentage change for a $5^{\circ}$ increase in rotation was calculated as (change per $5 \%$ absolute predicted value at zero degrees) x 100 .

\begin{tabular}{|c|c|c|c|c|c|c|c|c|}
\hline \multirow[b]{2}{*}{ Parameter } & & \multirow[b]{2}{*}{$\left(-10^{\circ}\right)$} & \multirow[b]{2}{*}{$\left(-5^{\circ}\right)$} & \multirow[b]{2}{*}{$\left(0^{\circ}\right)$} & \multirow[b]{2}{*}{$\left(5^{\circ}\right)$} & \multirow[b]{2}{*}{$\left(10^{\circ}\right)$} & \multicolumn{2}{|c|}{ Predicted change per $5^{\circ}$} \\
\hline & & & & & & & $\begin{array}{c}\text { Change } \\
\text { SE } \\
\text { P-value }\end{array}$ & Percentage \\
\hline \multirow{4}{*}{ B1 } & Mean & 17.01 & 17.70 & 18.24 & 18.78 & 19.31 & 0.567714 & \\
\hline & Mean Diff & -1.23 & -0.54 & 0.00 & 0.54 & 1.04 & 0.0547735 & 3.11 \\
\hline & SE Diff & 0.26 & 0.12 & 0.00 & 0.15 & 1.61 & $<0.001$ & \\
\hline & Mean & 17.38 & 16.87 & 16.47 & 16.09 & 15.61 & -0.432 & \\
\hline \multirow[t]{3}{*}{ B2 } & Mean Diff & 0.91 & 0.41 & 0.00 & -0.37 & -0.86 & 0.035153 & -2.62 \\
\hline & SE Diff & 0.20 & 0.10 & 0.00 & 0.09 & 0.21 & $<0.001$ & \\
\hline & Mean & 145.61 & 145.43 & 145.29 & 145.13 & 145.38 & -0.0754285 & \\
\hline \multirow[t]{3}{*}{ B3 } & Mean Diff & 0.32 & 0.14 & 0.00 & -0.16 & 0.09 & 0.057359 & -0.05 \\
\hline & SE Diff & 0.27 & 0.12 & 0.00 & 0.13 & 0.35 & 0.19 & \\
\hline & Mean & 5.19 & 4.92 & 4.73 & 4.32 & 3.91 & -0.3154285 & \\
\hline \multirow[t]{3}{*}{ B4 } & Mean Diff & 0.46 & 0.19 & 0.00 & -0.41 & -0.82 & 0.035773 & -6.67 \\
\hline & SE Diff & 0.11 & 0.05 & 0.00 & 0.13 & 0.20 & $<0.001$ & \\
\hline & Mean & 3.97 & 4.27 & 4.49 & 4.75 & 5.41 & 0.3355715 & \\
\hline \multirow[t]{3}{*}{ B5 } & Mean Diff & -0.52 & -0.22 & 0.00 & 0.26 & 0.92 & 0.042845 & 7.47 \\
\hline & SE Diff & 0.06 & 0.03 & 0.00 & 0.06 & 0.30 & $<0.001$ & \\
\hline & Mean & 2.12 & 2.35 & 2.42 & 2.55 & 2.86 & 0.1675715 & \\
\hline \multirow[t]{3}{*}{ B6 } & Mean Diff & -0.30 & -0.07 & 0.00 & 0.13 & 0.44 & 0.0177905 & 6.92 \\
\hline & SE Diff & 0.08 & 0.03 & 0.00 & 0.05 & 0.08 & $<0.001$ & \\
\hline & Mean & 36.38 & 36.17 & 35.66 & 35.48 & 34.80 & -0.3844285 & \\
\hline \multirow[t]{3}{*}{ B7 } & Mean Diff & 0.72 & 0.50 & 0.00 & -0.19 & -0.86 & 0.091552 & -1.08 \\
\hline & SE Diff & 0.58 & 0.41 & 0.00 & 0.19 & 0.44 & $<0.001$ & \\
\hline & Mean & 71.38 & 72.03 & 73.01 & 73.48 & 74.26 & 0.7214285 & \\
\hline \multirow[t]{3}{*}{ B8 } & Mean Diff & -1.63 & -0.97 & 0.00 & 0.47 & 1.25 & 0.091267 & 0.99 \\
\hline & SE Diff & 0.60 & 0.34 & 0.00 & 0.13 & 0.25 & $<0.001$ & \\
\hline & Mean & 72.24 & 71.80 & 71.33 & 71.04 & 70.63 & -0.397857 & \\
\hline \multirow[t]{2}{*}{ B9 } & Mean Diff & 0.92 & 0.47 & 0.00 & -0.28 & -0.70 & 0.0339335 & -0.56 \\
\hline & SE Diff & 0.13 & 0.12 & 0.00 & 0.09 & 0.17 & $<0.001$ & \\
\hline
\end{tabular}

angular parameters showed relatively more significant changes than the ratios. The range of $\pm 10^{\circ}$ of MCPJ rotation was considered to be the acceptable range of rotation to measure all the $27 \mathrm{MCPJ}$ parameters. The equation that was developed in this study can be used to estimate the deviation of the vertical angles from ZDP in future and archived radiographs. Further investigations about the effect of limb rotation around the horizontal axis while considering the effects of joint angle changes on MCPJ parameters are clearly required. 


\begin{tabular}{|c|c|c|c|c|c|c|c|c|}
\hline & Mean & 38.93 & 39.41 & 39.66 & 40.39 & 40.88 & 0.4894285 & \\
\hline \multirow[t]{3}{*}{ B11 } & Mean Diff & -0.73 & -0.25 & 0.00 & 0.74 & 1.23 & 0.07612 & 1.23 \\
\hline & SE Diff & 0.20 & 0.07 & 0.00 & 0.35 & 0.42 & $<0.001$ & \\
\hline & Mean & 39.02 & 38.68 & 38.21 & 37.75 & 37.34 & -0.428571 & \\
\hline \multirow[t]{3}{*}{ B13 } & Mean Diff & 0.81 & 0.47 & 0.00 & -0.46 & -0.87 & 0.049141 & -1.12 \\
\hline & SE Diff & 0.17 & 0.11 & 0.00 & 0.15 & 0.25 & $<0.001$ & \\
\hline & Mean & 102.05 & 101.91 & 102.13 & 101.85 & 101.78 & -0.060857 & \\
\hline \multirow[t]{3}{*}{ B14 } & Mean Diff & -0.08 & -0.22 & 0.00 & -0.28 & -0.36 & 0.0961815 & -0.06 \\
\hline & SE Diff & 0.26 & 0.11 & 0.00 & 0.38 & 0.56 & 0.53 & \\
\hline & Mean & 1.25 & 1.19 & 1.14 & 1.08 & 1.05 & -0.051 & \\
\hline \multirow[t]{3}{*}{ W2/W3 } & Mean Diff & 0.11 & 0.05 & 0.00 & -0.06 & -0.09 & 0.0021195 & -4.47 \\
\hline & SE Diff & 0.01 & 0.01 & 0.00 & 0.01 & 0.01 & $<0.001$ & \\
\hline & Mean & 0.95 & 0.97 & 0.98 & 1.00 & 1.01 & 0.014857 & \\
\hline \multirow[t]{3}{*}{ W4/W5 } & Mean Diff & -0.03 & -0.01 & 0.00 & 0.02 & 0.03 & 0.000973 & 1.51 \\
\hline & SE Diff & 0.00 & 0.00 & 0.00 & 0.01 & 0.01 & $<0.001$ & \\
\hline & Mean & 1.11 & 1.11 & 1.11 & 1.11 & 1.11 & -0.0005715 & \\
\hline \multirow[t]{3}{*}{ W1/W6 } & Mean Diff & 0.00 & 0.00 & 0.00 & 0.00 & 0.00 & 0.0005525 & -0.05 \\
\hline & SE Diff & 0.00 & 0.00 & 0.00 & 0.00 & 0.00 & 0.30 & \\
\hline & Mean & 0.97 & 0.94 & 0.91 & 0.88 & 0.85 & 0.030422 & \\
\hline \multirow[t]{3}{*}{ W4/W7 } & Mean Diff & 0.06 & 0.03 & 0.00 & -0.03 & -0.05 & 0.001815 & 3.48 \\
\hline & SE Diff & 0.01 & 0.01 & 0.00 & 0.01 & 0.01 & $<0.001$ & \\
\hline & Mean & 0.95 & 0.98 & 1.03 & 1.08 & 1.14 & -0.0472915 & \\
\hline \multirow[t]{3}{*}{ W5/W8 } & Mean Diff & -0.08 & -0.05 & 0.00 & 0.05 & 0.11 & 0.0022395 & -4.37 \\
\hline & SE Diff & 0.01 & 0.01 & 0.00 & 0.01 & 0.01 & $<0.001$ & \\
\hline & Mean & 0.48 & 0.48 & 0.48 & 0.48 & 0.48 & 0.001857 & \\
\hline \multirow[t]{3}{*}{ W4/(W7+W8) } & Mean Diff & 0.00 & 0.00 & 0.00 & 0.00 & 0.01 & 0.00065 & 0.39 \\
\hline & SE Diff & 0.00 & 0.00 & 0.00 & 0.00 & 0.00 & 0.0040 & \\
\hline & Mean & 0.50 & 0.49 & 0.49 & 0.48 & 0.48 & -0.005857 & \\
\hline \multirow[t]{3}{*}{ W5/(W7+W8) } & Mean Diff & 0.01 & 0.01 & 0.00 & -0.01 & -0.01 & 0.0006335 & -1.20 \\
\hline & SE Diff & 0.00 & 0.00 & 0.00 & 0.00 & 0.00 & $<0.001$ & \\
\hline & Mean & 1.28 & 1.20 & 1.12 & 1.04 & 1.00 & -0.071 & \\
\hline \multirow[t]{3}{*}{ W7/W8 } & Mean Diff & 0.16 & 0.08 & 0.00 & -0.07 & -0.12 & 0.0040035 & -6.36 \\
\hline & SE Diff & 0.02 & 0.01 & 0.00 & 0.01 & 0.02 & $<0.001$ & \\
\hline & Mean & 0.99 & 0.99 & 1.01 & 1.01 & 1.03 & 0.0104285 & \\
\hline \multirow[t]{3}{*}{ W2/W7 } & Mean Diff & -0.02 & -0.02 & 0.00 & 0.01 & 0.02 & 0.001551 & 1.04 \\
\hline & SE Diff & 0.01 & 0.01 & 0.00 & 0.00 & 0.01 & $<0.001$ & \\
\hline & Mean & 1.00 & 0.99 & 0.98 & 0.98 & 0.97 & -0.008 & \\
\hline \multirow[t]{3}{*}{ W3/W8 } & Mean Diff & 0.02 & 0.01 & 0.00 & -0.01 & -0.01 & 0.0017275 & -0.82 \\
\hline & SE Diff & 0.01 & 0.01 & 0.00 & 0.01 & 0.01 & $<0.001$ & \\
\hline & Mean & 0.57 & 0.55 & 0.54 & 0.52 & 0.51 & -0.0145715 & \\
\hline \multirow[t]{2}{*}{ W7/W6 } & Mean Diff & 0.03 & 0.02 & 0.00 & -0.02 & -0.03 & 0.0012635 & -2.72 \\
\hline & SE Diff & 0.01 & 0.00 & 0.00 & 0.00 & 0.01 & $<0.001$ & \\
\hline
\end{tabular}




\begin{tabular}{|c|c|c|c|c|c|c|c|c|}
\hline & Mean & 0.44 & 0.46 & 0.48 & 0.50 & 0.51 & 0.0172855 & \\
\hline \multirow[t]{3}{*}{ W8/W6 } & Mean Diff & -0.04 & -0.02 & 0.00 & 0.01 & 0.03 & 0.000944 & 3.57 \\
\hline & SE Diff & 0.00 & 0.00 & 0.00 & 0.00 & 0.00 & $<0.001$ & \\
\hline & Mean & 0.95 & 0.95 & 0.95 & 0.95 & 0.95 & -0.0184285 & \\
\hline \multirow[t]{3}{*}{$\mathrm{H} 1 / \mathrm{H} 2$} & Mean Diff & 0.00 & 0.00 & 0.00 & 0.00 & 0.00 & 0.010381 & -1.94 \\
\hline & SE Diff & 0.00 & 0.00 & 0.00 & 0.00 & 0.00 & 0.076 & \\
\hline & Mean & 0.51 & 0.51 & 0.51 & 0.51 & 0.51 & 0.0007145 & \\
\hline \multirow[t]{3}{*}{ H1/H3 } & Mean Diff & 0.00 & 0.00 & 0.00 & 0.00 & 0.00 & 0.000295 & 0.14 \\
\hline & SE Diff & 0.00 & 0.00 & 0.00 & 0.00 & 0.00 & 0.015 & \\
\hline & Mean & 0.52 & 0.52 & 0.54 & 0.54 & 0.54 & 0.0084285 & \\
\hline \multirow[t]{2}{*}{$\mathrm{H} 2 / \mathrm{H} 3$} & Mean Diff & -0.03 & -0.03 & 0.00 & 0.00 & 0.00 & 0.004426 & 1.55 \\
\hline & SE Diff & 0.03 & 0.03 & 0.00 & 0.00 & 0.00 & 0.057 & \\
\hline
\end{tabular}

Table 2. Means of changes from the zero degrees, regardless of whether they increased or decreased, in MCPJ rotation around the vertical axis for all parameters.

\begin{tabular}{|c|c|c|c|c|}
\hline Category of parameters & Parameters & 0 & $\pm 5^{\circ}$ & $\pm 10^{\circ}$ \\
\hline \multirow{11}{*}{ Angular parameters } & B1 & 0 & 0.567714 & 1.135428 \\
\hline & B2 & 0 & -0.432 & -0.864 \\
\hline & B3 & 0 & -0.0754285 & -0.150857 \\
\hline & B4 & 0 & -0.3154285 & -0.630857 \\
\hline & B5 & 0 & 0.3355715 & 0.671143 \\
\hline & B6 & 0 & 0.1675715 & 0.335143 \\
\hline & B7 & 0 & -0.3844285 & -0.768857 \\
\hline & B8 & 0 & 0.7214285 & 1.442857 \\
\hline & B9 & 0 & -0.397857 & -0.795714 \\
\hline & B11 & 0 & 0.4894285 & 0.978857 \\
\hline & $\begin{array}{l}\text { B13 } \\
\text { B14 }\end{array}$ & $\begin{array}{l}0 \\
0\end{array}$ & $\begin{array}{l}-0.428571 \\
-0.060857\end{array}$ & $\begin{array}{l}-0.857142 \\
-0.121714\end{array}$ \\
\hline \multirow{15}{*}{ Ratio parameters } & $\mathrm{W} 2 / \mathrm{W} 3$ & 0 & -0.051 & -0.102 \\
\hline & W4/W5 & 0 & 0.014857 & 0.029714 \\
\hline & W1/W6 & 0 & -0.0005715 & -0.001143 \\
\hline & W4/W7 & 0 & 0.030422 & 0.060844 \\
\hline & W5/W8 & 0 & -0.0472915 & -0.094583 \\
\hline & $\mathrm{W} 4 /(\mathrm{W} 7+\mathrm{W} 8)$ & 0 & 0.001857 & 0.003714 \\
\hline & $\mathrm{W} 5 /(\mathrm{W} 7+\mathrm{W} 8)$ & 0 & -0.005857 & -0.011714 \\
\hline & W7/W8 & 0 & -0.071 & -0.142 \\
\hline & W2/W7 & 0 & 0.0104285 & 0.020857 \\
\hline & W3/W8 & 0 & -0.008 & -0.016 \\
\hline & W7/W6 & 0 & -0.0145715 & -0.029143 \\
\hline & W8/W6 & 0 & 0.0172855 & 0.034571 \\
\hline & $\mathrm{H} 1 / \mathrm{H} 2$ & 0 & -0.0184285 & -0.036857 \\
\hline & $\mathrm{H} 1 / \mathrm{H} 3$ & 0 & 0.0007145 & 0.001429 \\
\hline & $\mathrm{H} 2 / \mathrm{H} 3$ & 0 & 0.0084285 & 0.016857 \\
\hline
\end{tabular}




\section{Acknowledgments}

The authors would like to thank the Libyan government for financial support, Colin Burvill and Jonathan Merritt for their help in designing and constructing the load cell, Loryn Anderson and Kane Wilson for their help in the scanning procedure and would also like to thank Garry Anderson for advice on statistical analysis.

\section{Conflict of interest}

Authors declare that there is no conflict of interest

\section{Ethical Approval}

As the experimental work conducted in cadavers, no ethical approved was required

Authors' contribution

Adulrhman Alrtib performed the experimental work and writing the manuscript; Aiman $\mathrm{H}$. Oheida and Mohamed $\mathrm{H}$. Abushhiwa revised the manuscript; Helen M.S. Davies designed the experiment, supervised the work and revised the manuscript. All authors contributed equally in the manuscript writing

5. References

[1] Curry TS, Dowdey JE, Murry RC, and Christensen EE, 1990: Christensen's physics of diagnostic radiology. Lippincott Williams \& Wilkins.

[2] Major PW, Johnson DE, Hesse KL, and Glover KE, 1994: Landmark identification error in posterior anterior cephalometrics. Angle Orthod, 64, 447-454. https://doi.org/10.1043/00033219(1994)064<0447:LIEIPA>2.0.CO;2

[3] Han CM, and Hurd CD, 2005: Practical diagnostic imaging for the veterinary technician. Elsevier/Mosby.

[4] Lavin LM, 2007: Radiography in veterinary technology / Lisa M. Lavin.4th ed. edn. St. Louis, Mo.: Saunders/Elsevier.

[5] Walter $L$, and Davies HM, 2001: Analysis of a radiographic technique for measurement of equine metacarpal bone shape. Equine Vet J Suppl, 141-144. https://doi.org/10.1111/j.2042-3306.2001.tb05377.x

[6] O'brien, TR, 2005: O'Brien's radiology for the ambulatory equine practitioner. Teton New Media.

[7] Major PW, Johnson DE, Hesse KL, and Glover KE, 1996: Effect of head orientation on posterior anterior cephalometric landmark identification. Angle Ortho., 66, 51-60. https://doi.org/ 10.1043/0003-3219(1996)066<0051:EOHOOP>2.3.CO;2.

[8] Yoon YJ, Kim DH, Yu PS, Kim HJ, Choi EH, and Kim KW, 2002: Effect of head rotation on posteroanterior cephalometric radiographs. Angle Orthod, 72, 36-42. https://doi.org/10.1043/00033219(2002)072<0036:EOHROP>2.0.CO;2

[9] Alrtib AM, Oheida AO, Abushhiwa MH, Davies HMS, 2019: Fetlock Parameters Development on Dorsopalmar Radiographs in the Equine Forelimb. Journal of Advanced Veterinary Research, 9, 49-55

[10] Alrtib AM, 2013: Radiographic bone morphometry of the equine metacarpophalangeal joint and relationships to the incidence of pathology in thoroughbred racehors. PhD Thesis. The University of Melbourne, Melbourne, Australia.

[11] Holmstrom M, Magnusson LE, Philipsson J, 1990: Variation in conformation of Swedish warmblood horses and conformationa characteristics of Elite sport horses. Equine Vet J, 22, 186-193. https://doi.org/10.1111/j.2042-3306. 1990.tb04245.x

[12] Weller R, Pfau T, May SA, Wilson AM, 2006: Variation in conformation in a cohort of National Hunt racehorses. Equine Vet J, 38, 616-621. https://doi.org/10.2746/042516406X150394

[13] Gregston MD, Kula T, Hardman P, Glaros A, and Kula K, 2004: A comparison of conventional and digital radiographic methods and cephalometric analysis software: I. hard tissue. In: Semin Orthod, 10. Elsevier.

[14] Kumar V, Ludlow J, Soares Cevidanes LH, and Mol A, 2008: In vivo comparison of conventional and cone beam CT synthesized cephalograms. Angle Orthod, 78, 873-879. https://doi.org/ 10.2319/082907-399.1

[15] Radtke K, Becher C, Noll Y, and Ostermeier S, 2010: Effect of limb rotation on radiographic alignment in total knee arthroplasties. Arch Orthop Trauma Surg, 130, 451-457. https://doi.org/ 10.1007/s00402009-0999-1

[16] Malkoc S, Sari Z, Usumez S, and Koyuturk AE, 2005: The effect of head rotation on cephalometric radiographs. Eur J Orthod, 27, 315321.https://doi.org/ 10.1093/ejo/cjh098

[17] Yoon YJ, Kim KS, Hwang MS, Kim HJ, Choi EH, and Kim KW, 2001: Effect of Head Rotation on Lateral Cephalometric Radiographs. Angle Orthod, 71, 396-403. https://doi.org/10.1043/00033219(2001)071<0396:EOHROL>2.0.CO;2

[18] Oheida, A. H., Anderson, G.A, Alrtib AM, Abushhiwa MH, Philip DJ, and Davies HMS 2017: Effect of Limb Rotation on Radiographic Measurements of Carpal Parameters in the Equine Carpus. J. Vet. Ad., 7, 1392-1402. 